\title{
Modular Theory and Geometry
}

\author{
B. Schroer and H.-W. Wiesbrock \\ FB Physik, FU Berlin, Arnimallee 14, Berlin 14195 \\ June, 1998
}

\begin{abstract}
In this letter we present some new results on modular theory and its application in quantum field theory. In doing this we develop some new proposals how to generalize concepts of geometrical action.

Therefore the spirit of this letter is more on a programmatic side with many details remaining to be elaborated.
\end{abstract}

\section{Introduction}

The basis of this new structure in QFT is modular theory. Mathematically it is a vast generalization of the modular factor which accounts for the difference between right and left Haar measure in the case of non unimodular groups as e.g. the group of upper triangle matrices. Tomita and later Takesaki succeeded to convert this idea into a powerful tool for the investigation of von Neumann algebras, see [Ta]. In fact Alain Connes could not have carried out his pathbreaking work on the classification of factor algebras without this theory. Modular theory is also looming behind the subfactor theory of Vaughn Jones. The physics side is equally impressive. At the time when Tomita presented his theory, Haag Hugenholz and Winnink published their fundamental work on (heat bath) thermal aspects of QFT, see [H/H/W]. The KMS condition (a name which they coined) was used up to that time by various physicist (in particular Kubo, Martin and Schwinger) as a clever mathematical trick in order to avoid to compute cumbersome traces in evaluating Gibbs thermal ensembles. In the hands of Haag Hugenholz and Winnink this formula became the key for their formulation 
of equilibrium quantum statistical mechanics directly in the thermodynamic limit for which the Gibbs representation becomes meaningless. The generator for the modular operator turns out to be a thermal hamiltonian with two-sided spectrum and finite fluctuations which acts on the original algebra as well as on its commutant which represents a kind of shadow world (which corresponds in the above analogy to group theory to the conjugate action ) Haa]. (See [Ja for new results on the physical interpretation of the underlying shadow world.) The Tomita antiunitary involution J turns out to be the normalized flip operation which maps the left into the right action. Later Bisognano and Wichmann [Bi/Wi] found another very nontrivial field theoretic illustration of the Tomita-Takesaki theory in their study of the algebra which is generated by fields restricted to the wedge region (i.e. the same (Rindler) region which played an important role in Unruh's discussion of the Hawking effect). In their case the modular group turned out to be the wedge-affiliated Lorentz boost and the Tomita $J$ was (up to a 90 degree rotation around the boost direction) the field theoretical TCP operator. Later Sewell showed that this beautiful result of Bisoganano and Wichmann might be seen as a generalization of the Unruh effect, see $\mathrm{Se}, \mathrm{Un}$. (Below we present an algebraic criterion which implies this property, see also [Bo3]) This time the shadow-world (the von Neumann commutant) was part of the real world namely the algebra of the causally disjoint region behind the wedge horizon. Besides having a thermal meaning the modular theory got a beautiful geometrical interpretation. This work (as well as some special prior observations on free fields $\mathrm{O} / \mathrm{L} / \mathrm{R}$ ) strongly suggested that there was a deep relation between modular theory and relativistic causality and localization. In more recent times Borchers [Bo1] and one of the present authors (H-W. W.) Wi2 turned the geometrical action of modular theory around and showed that with two subalgebras in appropriate modular position one can build up spacetime symmetries $(\mathrm{d}=1+1$ conformal $\mathrm{G} / \mathrm{L} / \mathrm{W}$ and, with somewhat stronger assumptions, also the higher dimensional cases [Ka/Wil Wi4]). Starting from Wigner's representation theory of positive energy representations it is possible to construct the unique net associated with the $(\mathrm{m}, \mathrm{s}=$ semiinteger) Wigner representation without using (nonunique) field coordinates as the generators of local algebras [Schr1].

We believe that these deep relation between space-time symmetry and pure quantum physical properties will be important for the understanding of the still evasive "Quantum Gravity". The modular structure also promises to clarify some points concerning the physics of the Wightman domain proper- 
ties [Schr1]. In fact the modular groups act linearity on the "field space" i. e. the space generated by applying a local field on the vacuum. Therefore this space, which is highly reducible under the Poincaré group, may according to a conjecture of Fredenhagen (based on the results in [Fr/Jod) in fact carry an irreducible representation of the union of all modular groups (an infinite dimensional group $\mathcal{G}_{\text {mod }}$ which contains in particular all local spacetime symmetries). The equivalence of fields with carriers of irreducible representations of an universal $\mathcal{G}_{\text {mod }}$ would add a significant conceptual element to LQP and give the notion of quantum fields a deep role which goes much beyond that of being simply generators of local algebras. Our arguments suggest that in chiral conformal QFT $\mathcal{G}_{\text {mod }}$ includes all local diffeomorphism. A related group theoretical approach this time starting from properties of modular involutions ("The Condition of Geometric Modular Action") was proposed in B/D/F/S . One obtains directly transformation groups on the indexed sets of the net. All these true QFT properties remain invisible in any quantization approach. Combining modular theory with scattering theory, the actual $J$ together with the incoming $J^{i n}$ can be used to obtain a new framework for nonperturbative interactions Schr1]. This last topic will be treated in a separate paper by the present two authors.

Besides the old result of Bisognano/Wichmann there are nowadays other interesting 'geometrical' actions of modular theory in chiral theories, see Bo/Yng . We present some new examples in chiral and higher dimensional models and give some outlook to future investigations of geometrical actions.

\section{The Bisognano-Wichmann Property}

We will start with the famous result of Bisognano/Wichmann $[\mathrm{Bi} / \mathrm{Wi}$. We will present a purely algebraic proof of that property. ${ }^{1}$

Let us first introduce some notations. We assume given a Poincaré covariant local net of observables which fulfills essential duality ( wedge duality). Let $U$ denote the representation of the Poincaré' group on the vacuum Hilbert space $\mathcal{H}, \Omega$ the vacuum vector. Let $l_{1}$ and $l_{2}$ be linear independent lightlike vectors and $W\left[l_{1} l_{2}\right]$ the wedge

$$
W\left[l_{1}, l_{2}\right]=\left\{\vec{x} \in R^{1,3} \mid \quad \vec{x}=\alpha l_{1}+\beta l_{2}+l^{\perp},\right. \text { with }
$$

\footnotetext{
${ }^{1}$ After finishing this part we were informed by H.-J. Borchers of a recent result by himself, Bo3. Below we compare both approaches.
} 


$$
\left.\alpha>0, \beta<0,\left(l^{\perp}, l_{i}\right)=\left(l^{\perp}, l_{j}\right)=0\right\}
$$

$\Lambda_{12}(t)$ the Lorentz boost to that wedge, i.e. the boosts which map the wedge $W\left[l_{1}, l_{2}\right]$ onto itself.

Let $\mathcal{A}\left(W\left[l_{1}, l_{2}\right]\right)$ be the observable algebra associated to the wedge. The Reeh-Schlieder property of the vacuum states that $\Omega$ is cyclic and separating for this algebra. We denote the associated modular objects by $\Delta_{12}, J_{12}$.

Before continuing with the results let us remind the reader on an observation by Borchers Bo4.

Assume wedge duality for the underlying net. Then we have

$$
\Delta_{12}^{i t}=F_{12}(t) U\left(\Lambda_{12}(-2 \pi t)\right)
$$

with some unitary group $F_{12}(t)$. Moreover modular theory gives

$$
\left[F_{12}(t), J_{12}\right]=\left[F_{12}(t), \Delta_{12}^{i s}\right]=\left[F_{12}(t), U\left(\Lambda_{12}(-2 \pi s)\right)\right]=0
$$

( $F_{12}$ leaves invariant $\mathcal{A}\left(W\left[l_{1}, l_{2}\right]\right)$ and $\Omega$.) Due to Borchers' result Bo1, Bo5 we immediately conclude

$$
\left[F_{12}(t), U(\text { translations })\right]=0,
$$

see Bo4]. Moreover there is a strongly dense set of elements $A \in \mathcal{A}\left(W\left[l_{1}, l_{2}\right]\right)$ s.t. $U\left(\Lambda_{1,2}(2 \pi t)\right) A \Omega, A \in \mathcal{A}\left(W\left[l_{1}, l_{2}\right]\right)$ can be analytically be continued to $t \in S\left(\frac{-1}{2}, 0\right)=\left\{z \in \mathbf{C} /-\frac{1}{2}<\operatorname{Im} z<0\right\}$..

So far Borchers' results. Let us come to an observation which one of the authors made in discussion with D. Guido:

Theorem 1 (Guido/Wiesbrock)

Additionally to the above we make the assumption

$$
\left(A \Omega \longrightarrow U\left(\Lambda_{1,2}\left(-\frac{i}{2}\right)\right) A^{*} \Omega, \quad A \in \mathcal{A}\left(W\left[l_{1}, l_{2}\right]\right)\right)
$$

is uniformly bounded.

Then the Bisognano-Wichmann Property holds for the net.

Proof. Let $F_{12}(t)=e^{i t f_{12}}$ be the unitary group above. From the assumption it follows

$f_{12}$ is semibounded. 
Now $F_{12}$ leaves invariant $\mathcal{A}\left(W\left[l_{1}, l_{2}\right]\right)$ which implies by a result of Borchers, $[\mathrm{Pd}]$, innerness of $F_{12}$, i.e.

$\exists e^{i t f} \in \mathcal{A}\left(W\left[l_{1}, l_{2}\right]\right)$ with

$$
e^{i t f} e^{-i t f_{12}} \in \mathcal{A}\left(W\left[l_{1}, l_{2}\right]\right)^{\prime} .
$$

$J_{12}$-invariance of $F_{12}$ implies $\mathrm{Ad} e^{i t f_{12}}=\mathrm{Ad} J_{12} e^{i t f_{12}} J_{12}$ and therefore

$$
J_{12} e^{i t f} e^{-i t f_{12}} J_{12}=e^{i \lambda} e^{i t f}, \lambda \in \mathbf{R}
$$

because $\mathcal{A}\left(W\left[l_{1}, l_{2}\right]\right)$ is a factor. Putting together we conclude

$$
e^{i t f_{12}}=J_{12} e^{i t f} J_{12} e^{i t f}
$$

$e^{i t f_{12}}$ is $\Delta_{12}$ invariant and therefore $e^{i t f}$ too. Similarly $e^{i t f}$ is $U\left(\Lambda_{12}\right)$ invariant and translation invariant along the baseline of the wedge.

But due to the cluster property the later implies $e^{i t f}=$ const.

We now make a comparison with the work of Borchers [Bo3].

As an algebraic criterion for the Bisognano-Wichmann Property, Borchers proposed a so called reality condition as follows:

Let $A \in W\left[l_{1}, l_{2}\right]$, such that $U\left(\Lambda_{1,2}(2 \pi t)\right) A^{*} \Omega$ can analytically be con-

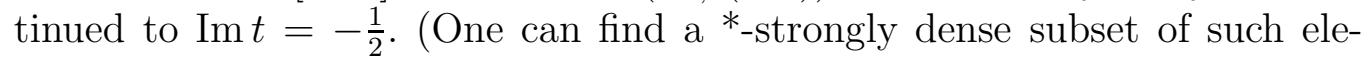
ments, see Bo4]) Then there exists $\widehat{A}$ and $\widetilde{A}$ affiliated with $\mathcal{A}\left(W\left[l_{1}, l_{2}\right]\right)^{\prime}=$ $\mathcal{A}\left(W\left[l_{2}, l_{1}\right]\right)$ with are related by:

$$
\widehat{A} \Omega=U\left(\Lambda_{1,2}(-i \pi)\right) A \Omega, \quad \widetilde{A} \Omega=U\left(\Lambda_{1,2}(-i \pi)\right) A^{*} \Omega .
$$

The reality condition states now:

For such $A$ one has $\widehat{A}^{*}=\widetilde{A}$, i.e. $U\left(\Lambda_{1,2}(-i \pi)\right) A^{*} \Omega=\widehat{A}^{*} \Omega$.

Rewritten in terms of the modular data this means:

$$
U\left(\Lambda_{1,2}(-i \pi)\right) S A \Omega=S^{*} U\left(\Lambda_{1,2}(-i \pi)\right) A \Omega
$$

for a *-strongly dense subset of $\mathcal{A}\left(W\left[l_{1}, l_{2}\right]\right)$, where $S$ denotes the Tomita conjugation to $\left(\mathcal{A}\left(W\left[l_{1}, l_{2}\right]\right), \Omega\right)$.

Now due to commutativity

$$
T:=U\left(\Lambda_{1,2}(-i \pi)\right) \Delta^{-\frac{1}{2}}=\Delta^{-\frac{1}{2}} U\left(\Lambda_{1,2}(-i \pi)\right)
$$


is a s. a. operator and the reality condition can be rephrased as

$$
J T J=T .
$$

But modular theory tells us that

$$
J U\left(\Lambda_{1,2}(-i \pi)\right) J=U\left(\Lambda_{1,2}(i \pi)\right), \quad J \Delta^{-\frac{1}{2}} J=\Delta^{\frac{1}{2}}
$$

which implies $J T J=T^{-1}$ and therefore

$$
T T^{*}=T^{2}=T J T J=T T^{-1}=1 .
$$

Therefore the reality condition gives boundedness of $T$ by 1 which implies the assumption of our theorem.

We don't see a natural physical motivation for our boundedness assumption. But the results suggest that one should look at a purely algebraic criterion which guarantees the Bisognano-Wichmann property. This would not rely on some generating fields which in the algebraic approach to quantum field theory play the role of a special choice of coordinating the physical system.

\section{On the modular theory of disjoint intervals}

In this section we present a modular interpretation of conformal diffeomorphisms.

\subsection{The Virasoro-algebra}

We consider the $U(1)$ - current algebra on the circle. Then we have an action

of the Virasoro-algebra on the physical vacuum Hilbert space of our model. The vacuum is defined as the unique invariant vector

$$
L_{1} \Omega=L_{-1} \Omega=L_{0} \Omega=0 .
$$

The usual commutation relations are:

$$
\left[L_{n}, L_{m}\right]=(n+m) L_{n+m}+\frac{1}{12}\left(m^{2}-1\right) m \delta_{m, n}
$$

so that:

$$
\left[L_{ \pm 2}, L_{0}\right]= \pm 2 L_{ \pm 2}, \quad\left[L_{2}, l_{-2}\right]=4 L_{0}+\frac{1}{2}
$$


holds. Then a simple computation shows

$$
\begin{aligned}
L_{0} & \mapsto \frac{1}{2} L_{0}+\frac{1}{16} \\
L_{1} & \mapsto \frac{1}{2 \sqrt{2}} L_{2} \\
L_{-1} & \mapsto \frac{1}{2 \sqrt{2}} L_{-2}
\end{aligned}
$$

gives an isomorphism of $s l(2 \mathbf{R})$ - Lie algebras. This second representation belongs to a $3-$ dim. subgroup in Diff $S^{1}$ given as follows.

$$
z \mapsto \sqrt{\frac{a+b z^{2}}{c+d z^{2}}} \quad\left(\begin{array}{ll}
a & b \\
c & d
\end{array}\right) \in S U(1,1)
$$

One notices the following (after discussion with M. Schmidt):

- $S U(1,1)$ maps the circle into itself

- the poles of $\frac{a+b z^{2}}{c+d z^{2}}$ lie outside the circle, the zeros inside

- Choose the cuts by adjoining both zeroes and both poles. In that way we get well defined maps.

- We get a representation of a twofold covering of the Möbiusgroup.

Geometrically the associated dilations leave invariant disjoint intervals of the following type: Given an interval, look at the square root of it elements. We get a disjoint union of intervals as the inverse image. Both parts separately are left invariant under that group. Therefore, if the modular groups act like dilations as above, we do not have Reeh-Schlieder Property for an arbitrary local algebra because of Takesaki's result, see [Stra]. I. e. this would either imply equality of the algebra of a small interval and the algebra associated to appropriate disjoint intervals or that the algebra is not cyclic.

But notice that due to the geometry it never happens that one part of such a region covers both parts of another type of such disjoint intervals.. Especially we do not get into conflict with additivity of the net and locality! This is the reason that such a modular theory is conceptually allowed. Next we show that this also happens. 


\subsection{Some Formulas}

Let us collect some well known formulas which help to understand the following computations. In the $S l(2 \mathbf{R})$ group we have the generator $D$ for the dilations, $P$ and $K$ for the translations resp. conformal translations. They are related to the Virasoro group by

$$
\begin{array}{rlrl}
L_{0} & =\frac{1}{2}(P+K) & D & =\frac{1}{2}\left(L_{1}-L_{-1}\right) \\
L_{1} & =D+\frac{1}{2}(P-K) & P & =\frac{1}{2}\left(L_{1}+L_{-1}\right)+L_{0} \\
L_{-1} & =D-\frac{1}{2}(P-K) & K & K=\frac{1}{2}\left(L_{-1}+L_{1}\right)-L_{0}
\end{array}
$$

It turns out to be helpful to switch from the compact (circle) picture to the noncompact on the reals.

$$
\begin{array}{lll}
\text { compact picture } & \rightarrow \text { non compact picture: } & z \mapsto-i \frac{z-1}{z+1} \\
\text { non compact picture } & \rightarrow \text { compact picture: } & x \mapsto \frac{i x+1}{-i x+1} .
\end{array}
$$

Under these transformation the relevant forms and the $L_{n}$ are mapped to the following.

$$
\begin{aligned}
d x & =-i \frac{2}{(z+i)^{2}} d z, \quad d z=2 i \frac{1}{(-i x+1)^{2}} d x \\
L_{n} & =z^{n+1} \frac{d}{d z}, \quad L_{n}=\frac{(-i x+1)^{2}}{2 i}\left(\frac{i x+1}{-i x+1}\right)^{n+1} \frac{d}{d x}
\end{aligned}
$$

A formal computation now shows:

$$
\begin{aligned}
z^{2} \circ L_{n} \circ \sqrt{z}=\frac{1}{2} L_{2 n}, \text { i.e. : } & \\
L_{ \pm 1} & \mapsto \frac{1}{2} L_{ \pm 2} \\
L_{0} & \mapsto \frac{1}{2} L_{0}
\end{aligned}
$$

in the non compact picture:

$$
\begin{aligned}
& z \quad \mapsto \quad z^{2} \simeq x \mapsto \frac{2 x}{1-x^{2}} \\
& z \quad \mapsto \sqrt{z} \simeq x \mapsto-\frac{1}{x}+\operatorname{sign}(x) \sqrt{1+\left(\frac{1}{x}\right)^{2}}
\end{aligned}
$$


In the non compact and compact picture it is easily seen that diffeomorphisms act symplectically:

$$
\omega(g, f)=\frac{1}{2} \int g(x) d f(x), \quad \omega(g, f)=\frac{1}{2} \int g(z) d f(z)
$$

\subsection{Transformation and Invariant Scalar Product}

Let $\left(\begin{array}{ll}a & b \\ c & d\end{array}\right) \in S l(2 \mathbf{R})$, then the underlying symmetry action is:

$$
x \mapsto-\frac{2 c x+d\left(1-x^{2}\right)}{2 a x+b\left(1-x^{2}\right)}+\operatorname{sign}(x) \sqrt{1+\left(-\frac{2 c x+d\left(1-x^{2}\right)}{2 a x+b\left(1-x^{2}\right)}\right)^{2}} .
$$

Moreover

$$
<f, g>=\int \frac{f(x) g(y)}{((x-y)(1+x y)+i 0)^{2}}\left(1+x^{2}\right)\left(1+y^{2}\right) d x d y
$$

gives an invariant scalar product.

proof:

Denote $\Gamma(x)=-\frac{1}{x}+\operatorname{sign}(x) \sqrt{1+\left(\frac{1}{x}\right)^{2}}$ then we compute

$$
\begin{aligned}
& \int \frac{f \circ \Gamma\left(\frac{a\left(\frac{2 x}{1-x^{2}}\right)+b}{c\left(\frac{2 x}{1-x^{2}}\right)+d}\right) g \circ \Gamma\left(\frac{a\left(\frac{2 y}{1-y^{2}}\right)+b}{c\left(\frac{2 y}{1-y^{2}}\right)+d}\right)}{((x-y)(1+x y)+i 0)^{2}}\left(1+x^{2}\right)\left(1+y^{2}\right) d x d y \\
= & \int \frac{f \circ \Gamma\left(\frac{a\left(\frac{2 x}{1-x^{2}}\right)+b}{c\left(\frac{2 x}{1-x^{2}}\right)+d}\right) g \circ \Gamma\left(\frac{a\left(\frac{2 y}{1-y^{2}}\right)+b}{c\left(\frac{2 y}{1-y^{2}}\right)+d}\right)}{\left(\frac{2 x}{1-x^{2}}-\frac{2 y}{1-y^{2}}+i 0\right)^{2}} \frac{2\left(1+x^{2}\right) 2\left(1+y^{2}\right)}{\left(1-x^{2}\right)^{2}\left(1-y^{2}\right)^{2}} d x d y
\end{aligned}
$$

One notices $d\left(\frac{2 x}{1-x^{2}}\right)=\frac{2\left(1+x^{2}\right)}{\left(1-x^{2}\right)^{2}}$ and thereby:

$$
\begin{gathered}
=\int \frac{f \circ \Gamma\left(\frac{a\left(\frac{2 x}{1-x^{2}}\right)+b}{c\left(\frac{2 x}{1-x^{2}}\right)+d}\right) g \circ \Gamma\left(\frac{a\left(\frac{2 y}{1-y^{2}}\right)+b}{c\left(\frac{2 y}{1-y^{2}}\right)+d}\right)}{\left(\frac{2 x}{1-x^{2}}-\frac{2 y}{1-y^{2}}+i 0\right)^{2}} d\left(\frac{2 x}{1-x^{2}}\right) d\left(\frac{2 y}{1-y^{2}}\right) \\
=\int \frac{f \circ \Gamma\left(\frac{2 x}{1-x^{2}}\right) g \circ \Gamma\left(\frac{2 y}{1-y^{2}}\right)}{\left(\frac{2 x}{1-x^{2}}-\frac{2 y}{1-y^{2}}+i 0\right)^{2}} d\left(\frac{2 x}{1-x^{2}}\right) d\left(\frac{2 y}{1-y^{2}}\right) \\
=\int \frac{f(x) g(y)}{((x-y)(1+x y)+i 0)^{2}}\left(1+x^{2}\right)\left(1+y^{2}\right) d x d y
\end{gathered}
$$


where the last equality follows from a substitution with $\left(x \mapsto \frac{2 x}{1-x^{2}}\right)$, which establishes invariance. qed.

The form is positive definite: Write $f=f \circ \Gamma \circ\left(\frac{2 x}{1-x^{2}}\right)$.

Define

$$
I(f)(x)=\int f(y) K_{I}(x, y) d y
$$

with

$$
k_{I}(x, y)=\frac{-1}{(x-y)}-\frac{1}{y} \frac{1}{(1+x y)}=\frac{-x\left(1+y^{2}\right)}{y(x-y)(1+x y)} .
$$

Then one computes:

$$
\frac{d}{d x} k_{I}(x, y)=\frac{\left(1+x^{2}\right)\left(1+y^{2}\right)}{[(x-y)(1+x y)]^{2}}=\frac{-1}{(x-y)^{2}}-\frac{1}{(1+x y)^{2}} .
$$

and we get:

$$
\omega(I f, g)=<f, g>=-\omega(f, I g) .
$$

Next we show that this scalar product gives us a Fock state on the Weyl algebra:

Denote

$$
\Gamma(f)(x):=f\left(\frac{x}{2}+\operatorname{sign}(x) \sqrt{1+\left(\frac{x}{2}\right)^{2}}\right)
$$

and

$$
I_{0}(f)(x)=\int \frac{-1}{(x-y+i 0)} f(y) d y
$$

which gives the usual vacuum Fock state of the theory Yng.

$$
\langle f, g\rangle_{0}=\omega\left(I_{0} f, g\right)=\int \frac{f(x) g(y)}{(x-y+i 0)^{2}} d x d y
$$

Then we compute:

$$
\begin{aligned}
\Gamma^{-1} \circ I_{0} \circ \Gamma(f)(x) & =\int \frac{-1}{\left(-\frac{1}{x}+x-y+i 0\right)} f\left(\frac{y}{2}+\operatorname{sign}(x) \sqrt{1+\left(\frac{y}{2}\right)^{2}}\right)(32) \\
& =\int \frac{-1}{\left(-\frac{1}{x}+x-\left(-\frac{1}{z}+z\right)+i 0\right)} f(z) \frac{z^{2}+1}{z^{2}} d z \\
& =\int \frac{-x\left(z^{2}+1\right)}{z^{2}\left(-1+x^{2}+\frac{x}{z}-z x+i 0\right)} f(z) d z
\end{aligned}
$$




$$
\begin{aligned}
& =\int \frac{-x\left(z^{2}+1\right)}{z\left(-z+x^{2} z+x-z^{2} x+i 0\right)} f(z) d z \\
& =\int \frac{-x\left(z^{2}+1\right)}{z(-z+x+i 0)(1+x z)} f(z) d z=I(f)(x)
\end{aligned}
$$

( One has to take care of the $+i 0$-term?!), so we get:

$$
\Gamma^{-1} \circ I_{0} \circ \Gamma=I \text {. }
$$

As is well known $I_{0}^{2}=-1$, from which we immediately conclude $I^{2}=-1$, i.e. the invariant scalar product produces a Fock state on the Weyl algebra.

\subsection{The KMS-Condition}

Let us show the KMS-Condition. For this we first make the following simple observation:

$$
\begin{aligned}
& f \in C_{0}^{\infty}([0,1]) \Rightarrow \Gamma(f) \in C_{0}^{\infty}([0, \infty[) \\
& \left.\left.f \in C_{0}^{\infty}(]-\infty,-1\right]\right) \Rightarrow \Gamma(f) \in C_{0}^{\infty}([0, \infty[) .
\end{aligned}
$$

Denote $<,>_{0}$ the scalar product of the vacuum state, $<,>_{1}$ the one above. Then we show:

$$
\begin{gathered}
<\Gamma(f), \Gamma(g)>_{0}=<f, g>_{1}: \\
\int d x d y \frac{f\left(-\frac{1}{x}+\operatorname{sign}(x) \sqrt{1+\left(\frac{1}{x}\right)^{2}}\right) g\left(-\frac{1}{y}+\operatorname{sign}(x) \sqrt{1+\left(\frac{1}{y}\right)^{2}}\right)}{(x-y+i 0)^{2}} \\
=\int d z d w \frac{f(z) g(w)}{\left(\frac{2 z}{1-z^{2}}-\frac{2 w}{1-w^{2}}+i 0\right)^{2}} \frac{1+z^{2}}{\left(1-z^{2}\right)^{2}} \frac{1+w^{2}}{\left(1-w^{2}\right)^{2}} \\
=\frac{1}{2} \int d z d w \frac{f(z) g(w)}{(z-w+i 0)^{2}} \frac{\left(1+z^{2}\right)\left(1+w^{2}\right)}{(1+z w)^{2}}
\end{gathered}
$$

Let us also introduce the notion

$V(\lambda)(f)(x)=f(\lambda x), \quad U(\lambda)(f)(x)=f\left(\frac{1-x^{2}}{2 \lambda^{2}}+\operatorname{sign}(x) \sqrt{1+\left(\frac{1-x^{2}}{2 \lambda^{2}}\right)^{2}}\right)$

then, an easy computation shows

$$
\Gamma^{-1} \circ V(\lambda) \circ \Gamma=U(\lambda) .
$$


To prove the KMS-property for the disjoint interval $]-\infty,-1] \cup[0,1]$ w.r.t. $U$, we use the strategy as in Yng .

Let $f, g \in C_{0}^{\infty}([0,1])$. Then

$$
<U(\lambda) f, g>_{1}=<V(\lambda) \circ \Gamma(f), \Gamma(g)>_{0}
$$

Due to (3.1), $\Gamma(f), \Gamma(g) \in C_{0}^{\infty}([0, \infty[)$. J. Yngavson has shown in Yng that for such functions the expectation value with $V(\lambda)$ can analytically be continued to $\operatorname{Im} \lambda=2 \pi$ with specific boundary values. This gives in our case:

$$
\begin{aligned}
\lim _{\theta \uparrow 2 \pi} & <U(\lambda) f, g>_{1}=\lim _{\theta \uparrow 2 \pi}<V(\lambda) \circ \Gamma(f), \Gamma(g)>=\langle\Gamma(g), \Gamma(f)\rangle= \\
& =\langle g, f\rangle_{1}
\end{aligned}
$$

This shows that $U(\lambda)$ fulfils the KMS-condition for the Weyl-algebra to $[0,1]$.

By the invariance and relation (3.2) we immediately get the same result for $\left.\left.f, g \in C_{0}^{\infty}(]-\infty,-1\right]\right)$, resp. $\left.\left.f \in C_{0}^{\infty}(]-\infty,-1\right]\right), g \in C_{0}^{\infty}([0,1])$.and thereby get that $U$ gives the modular group to the disjoint intervals. The methods of Yng then also apply to give

$$
J=\Gamma^{-1} \circ J_{0} \circ \Gamma \Rightarrow J(f)(x)=f^{*}\left(\frac{1}{x}\right)
$$

The same methods should also work for the $L_{3,} L_{-3}$ case, where one has to use the third root. In this way we get an action of these other diffeomorphism groups as automorphisms on the local net. As is well known they can be implemented on the vacuum theory by Bogoliubov automorphisms.

We expect that these results hold in all loop group models as they were constructed for example in [Was].

The final upshot of this section is that several diffeomorphism actions can occur as the action of some modular groups of certain observable algebras w.r.t. to a proper state.

\section{Hidden Symmetry}

As was shown in $\mathrm{Bo} / \mathrm{Yng}$ it might happen that the modular group acts only geometrically on a certain subregion. We show how this comes up very naturally in higher dimensional situations. We start with some purely group theoretical observations on an interesting subgroup of the Poincaré group 
[Schr3]. The standard wedge situation suggests to decompose the Poincaré group generators into longitudinal, transversal and mixed generators

$$
P_{ \pm}=\frac{1}{\sqrt{2}}\left(P_{t} \pm P_{z}\right), M_{t z} ; M_{x y}, P_{i} ; G_{i}^{( \pm)} \equiv \frac{1}{\sqrt{2}}\left(M_{i t} \pm M_{i z}\right), i=x, y
$$

The generators $G_{i}^{( \pm)}$are precisely the "translational" pieces of the euclidean stability groups $E^{( \pm)}(2)$ of the two light vectors $e^{( \pm)}=(1,0,0, \pm 1)$ which appeared for the first time in Wigner's representation theory for zero mass particles. As one reads off from the commutation relations, $P_{i}, G_{i}^{(+)}, P_{ \pm}$have the interpretation of a central extension of a transversal "Galilei group" with the two "translations" $G_{i}^{(+)}$representing the Galilei generators, $P_{+}$the central "mass" and $P_{-}$the "nonrelativistic Hamiltonian". The longitudinal boost $M_{t z}$ scales the Galilei generators $G_{i}^{(+)}$and the "mass" $P_{+}$. Geometrically the $G_{i}^{(+)}$change the standard wedge (it tilts the longitudinal plane) and the corresponding finite transformations generate a family of wedges whose envelope is the half-space $x_{-} \geq 0$. The Galilei group together with the boost $M_{t z}$ generate an 8-parametric subgroup $G^{(+)}(8)$ inside the 10-parametric Poincaré group:

$$
G^{(+)}(8): P_{ \pm}, M_{t z} ; M_{x y}, P_{i} ; G_{i}^{(+)}
$$

The modular reflection $J$ transforms this group into an isomorphic $G^{(-)}(8)$. All observation have interesting generalizations to the conformal group in massless theories in which case the associated natural space-time region is the double cone. This subgroup is intimately related to the notion of "modular intersection". Let $l_{1}, l_{2}$ and $l_{3}$ be 3 linear independent light-like vectors and consider two wedges $W\left(l_{1}, l_{2}\right), W\left(l_{1}, l_{3}\right)$ with $\Lambda_{12}$ and $\Lambda_{13}$ the associated Lorentz boosts. As a result of this common $l_{1}$ the algebras $\mathcal{N}=$ $\mathcal{A}\left(W\left(l_{1}, l_{2}\right)\right), \mathcal{M}=\mathcal{A}\left(W\left(l_{1}, l_{3}\right)\right)$ have a modular intersection with respect to the vector $\Omega$. Especially $(\mathcal{N} \cap \mathcal{M}) \subset \mathcal{M}, \Omega)$ is a modular inclusion Wi1, Ar/Zs, Bo5. Identifying $W\left(l_{1}, l_{2}\right)$ with the above standard wedge, we notice that the longitudinal generators $P_{ \pm}, M_{t z}$ are related to the inclusion of the standard wedge algebra into the full algebra $B(H)$, whereas the Galilei generators $G_{i}^{(+)}$are the "translational" part of the stability group of the common light vector $l_{1}$ (i.e. of the Wigner light-like little group). These generators $G_{i}^{(+)}$should be considered as being associated with a common light ray shared by two wedges whereas all the other generators in (42) are either longitudinal or transversal to one wedge (the standard wedge). 
To simplify the situation let us take $\mathrm{d}=1+2$ with $G^{(+)}(4)$, in which case there is only one Galilei generator $G$. In addition to the "visible" geometric subgroup of the Poincaré group, the modular theory produces a "hiddensymmetry transformation which belongs to a region which is a intersection of two wedges.

Let us now consider the 3-dim. situation from the point of view of algebraic QFT. We assume the Bisognano-Wichmann property for the net and also assume that it is fulfills additivity. Denote $l_{1}, l_{2}, l_{3}$ three linear independent lightlike vectors and $W\left[l_{1}, l_{2}\right], W\left[l_{1}, l_{2}\right]$ two wedges characterized by their two lightlike vectors. Denote $\Lambda_{12}$ resp. $\Lambda_{13}$ the related Lorentz boosts. Due to their common light-ray $l_{1}$ the associated observable algebras have - modular intersection w.r.t. $\Omega$, see [Bo2, Wi4]. To simplify the notation we use $\mathcal{N}, \mathcal{M}$ for the algebras. Then $((\mathcal{N} \cap \mathcal{M}) \subset \mathcal{M}, \Omega)$ is a modular inclusion, see [Wi1, Ar/Zs, Bo2, Wi4 and

$$
U_{\mathcal{N} \cap \mathcal{M}, \mathcal{M}}(a):=\exp \left(\frac{i a}{2 \pi}\left(\ln \Delta_{N \cap M}-\ln \Delta_{M}\right)\right)
$$

is a unitary group with positive generator. Moreover one has

$$
\begin{gathered}
U_{\mathcal{N} \frown \mathcal{M}, \mathcal{M}}\left(1-e^{-2 \pi t}\right)=\Delta_{\mathcal{M}}^{i t} \Delta_{\mathcal{N} \frown \mathcal{M}}^{-i t} \\
U_{\mathcal{N} \cap \mathcal{M}, \mathcal{M}}\left(e^{-2 \pi t} a\right)=\Delta_{\mathcal{M}}^{i t} U_{\mathcal{N} \cap \mathcal{M}, \mathcal{M}}(a) \Delta_{\mathcal{M}}^{-i t} \\
A d U_{\mathcal{N} \cap \mathcal{M}, \mathcal{M}}(-1)(\mathcal{M})=\mathcal{N} \cap \mathcal{M}
\end{gathered}
$$

and

$$
J_{\mathcal{M}} U_{\mathcal{N} \frown \mathcal{M}, \mathcal{M}}(a) J_{\mathcal{M}}=U_{\mathcal{N} \frown \mathcal{M}, \mathcal{M}}(-a) .
$$

Similar results hold for $\mathcal{N}$ replacing $\mathcal{M}$, see [Bo2, Wi3]. Due to the intersection property we finally have the relation

$$
\left[U_{\mathcal{N} \frown \mathcal{M}, \mathcal{M}}(a), U_{\mathcal{N} \frown \mathcal{M}, \mathcal{N}}(b)\right]=0
$$

which enables one to define the unitary group

$$
U_{\mathcal{N} \frown \mathcal{M}}(a)=U_{\mathcal{N} \frown \mathcal{M}, \mathcal{M}}(-a) U_{\mathcal{N} \frown \mathcal{M}, \mathcal{N}}(a) .
$$

This later group can be rewritten as

$$
U_{\mathcal{N} \frown \mathcal{M}}\left(1-e^{-2 \pi t}\right)=\Delta_{\mathcal{M}}^{i t} \Delta_{\mathcal{N}}^{-i t}
$$


and thereby recognized to be in our physical application the 1-parameter Galilean subgroup $G(42)$ in the above remarks.

Now we notice that for $a<0$

$$
\begin{aligned}
A d U_{\mathcal{N} \frown \mathcal{M}, \mathcal{M}}(a)(\mathcal{M}) & =A d \Delta_{\mathcal{M}}^{-i\left(\frac{1}{2 \pi} \ln -a\right)} U_{\mathcal{N} \frown \mathcal{M}, \mathcal{M}}(-1)(\mathcal{M}) \\
& =A d \Delta_{\mathcal{M}}^{-i\left(\frac{1}{2 \pi} \ln -a\right)}(\mathcal{N} \cap \mathcal{M})
\end{aligned}
$$

Because $\Delta_{\mathcal{M}}^{i t}$ acts geometrically as Lorentz boosts, we fully know the geometrical action of $U_{\mathcal{N} \frown \mathcal{M}, \mathcal{M}}(a)$ on $\mathcal{M}$ for $a<0$. For $a>0$ we notice

$$
\begin{aligned}
\operatorname{Ad} U_{\mathcal{N} \frown \mathcal{M}, \mathcal{M}}(1)(\mathcal{M}) & =A d U_{\mathcal{N} \frown \mathcal{M}, \mathcal{M}}(2)(\mathcal{M} \cap \mathcal{N})=\operatorname{Ad} J_{\mathcal{M}} J_{\mathcal{N} \frown \mathcal{M}}(\mathcal{M} \cap(\mathcal{A} 9) \\
& =A d J_{\mathcal{M}}\left(\mathcal{M}^{\prime} \cup \mathcal{N}^{\prime}\right)
\end{aligned}
$$

and again, due to the geometrical action of $J_{M}$ we have a geometrical action on $\mathcal{M}$ for $a>0$.

$$
A d U_{\mathcal{N} \cap \mathcal{M}, \mathcal{M}}(a)(\mathcal{M})=A d \Delta_{\mathcal{M}}^{-i\left(\frac{1}{2 \pi} \ln a\right)} J_{\mathcal{M}}\left(\mathcal{M}^{\prime} \cup \mathcal{N}^{\prime}\right)
$$

From these observations and with $U_{\mathcal{N} \cap \mathcal{M}, \mathcal{M}}\left(1-e^{-2 \pi t}\right)=\Delta_{\mathcal{M}}^{i t} \Delta_{\mathcal{M} \cap \mathcal{N}}^{-i t}$ we get for $t<0$ :

$$
A d \Delta_{\mathcal{N} \cap \mathcal{M}}^{i t}(\mathcal{M})=A d \Delta_{\mathcal{M}}^{\left(-\frac{i}{2 \pi} \ln \left(e^{-2 \pi t}-1\right)\right)} J_{\mathcal{M}}\left(\mathcal{M}^{\prime} \cup \mathcal{N}^{\prime}\right)
$$

and in case of $t>0$ :

$$
A d \Delta_{\mathcal{N} \cap \mathcal{M}}^{i t}(\mathcal{M})=A d \Delta_{\mathcal{M}}^{\left(-\frac{i}{2 \pi} \ln \left(1-e^{-2 \pi t}\right)\right)}(\mathcal{N} \cap \mathcal{M})
$$

Similar results hold for $\mathcal{N}$ replacing $\mathcal{M}$. With the same methods we get:

$$
\begin{aligned}
A d \Delta_{\mathcal{N} \cap \mathcal{M}}^{i t} \Delta_{\mathcal{N}}^{i s}(\mathcal{M}) & =A d \Delta_{\mathcal{N} \cap \mathcal{M}}^{i t} \Delta_{\mathcal{N}}^{i s} \Delta_{\mathcal{M}}^{-i s}(\mathcal{M}) \\
& =A d \Delta_{\mathcal{N} \cap \mathcal{M}}^{i t} U_{\mathcal{M} \cap \mathcal{N}}\left(e^{-2 \pi s}-1\right)(\mathcal{M})
\end{aligned}
$$

where $U_{\mathcal{N} \cap \mathcal{M}}$ is the 1-parameter Lorentz subgroup (the Galilei subgroup $G$ in (42) associated with the modular intersection, see [Bo2, Wi4]. This gives:

$$
\begin{aligned}
A d \Delta_{\mathcal{N} \cap \mathcal{M}}^{i t} \Delta_{N}^{i s}(\mathcal{M}) & =A d U_{\mathcal{M} \cap \mathcal{N}}\left(e^{-2 \pi t}\left(e^{-2 \pi s}-1\right)\right) \Delta_{\mathcal{N} \cap \mathcal{M}}^{i t}(\mathcal{M}) \\
& =A d U_{\mathcal{M} \cap \mathcal{N}}\left(e^{-2 \pi t}\left(e^{-2 \pi s}-1\right)\right) \Delta_{\mathcal{M}}^{-\frac{1}{2 \pi} \ln \left(1-e^{-2 \pi t}\right)}(\mathcal{M} \cap \mathcal{N}),
\end{aligned}
$$

if $t>0$ and similar for $t<0$. Therefore we get a geometrical action of $\Delta_{\mathcal{N} \cap \mathcal{M}}^{i t}$ on $A d \Delta_{\mathcal{N}}^{i s}(\mathcal{M})$. 
A look at the proof shows that the essential ingredients are the special commutation relations. Due to

$$
\Delta_{\mathcal{M} \cap \mathcal{N}}^{i t}=\Delta_{\mathcal{M}}^{i t} U_{\mathcal{N} \cap \mathcal{M}, \mathcal{M}}\left(1-e^{-2 \pi t}\right)=\Delta_{\mathcal{M}}^{i t} J_{\mathcal{M}} U_{\mathcal{N} \cap \mathcal{M}, \mathcal{M}}\left(e^{-2 \pi t}-1\right) J_{\mathcal{M}}
$$

and the well established geometrical action of $\Delta_{\mathcal{M}}^{i t}$ and $J_{\mathcal{M}}$ it is enough to consider the action of $U_{\mathcal{N} \cap \mathcal{M}, \mathcal{M}}$ or similarly $U_{\mathcal{N} \cap \mathcal{M}, \mathcal{N}}$. For these groups we easily get

$$
A d U_{\mathcal{N} \cap \mathcal{M}, \mathcal{M}}(a) \Delta_{\mathcal{N}}^{i s} \Delta_{\mathcal{M}}^{-i t}(\mathcal{N})=A d \Delta_{\mathcal{N}}^{i s} \Delta_{\mathcal{M}}^{i t} U_{\mathcal{N} \cap \mathcal{M}, \mathcal{M}}\left(e^{-2 \pi(s+t)} a\right)(\mathcal{N})
$$

and due to the above remarks the geometrical action of $\Delta_{\mathcal{N} \cap \mathcal{M}}^{i t}$ on the algebras of the type $A d \Delta_{\mathcal{N}}^{i s} \Delta_{\mathcal{M}}^{-i t}(\mathcal{M})$.

Now, the lightlike translations $U_{\text {transl }_{1}}(a)$ in $l_{1}$ direction fulfill the positive spectrum condition and map $\mathcal{N} \cap \mathcal{M}$ into itself for $a>0$. Therefore we have the Borchers commutator relations with $\Delta_{\mathcal{M} \cap \mathcal{N}}^{i t}$ and get

$$
A d \Delta_{\mathcal{N} \frown \mathcal{M}}^{i t} U_{\text {transl }_{1}}(a)(\mathcal{M})=A d U_{\text {transl }_{1}}\left(e^{-2 \pi t} a\right) \Delta_{\mathcal{N} \frown \mathcal{M}}^{i t}(\mathcal{M})
$$

The additivity of the net tells us that taking unions of the algebra corresponds to the causal unions of localization regions. The assumed duality allows us to pass to causal complements and thereby to intersections of the underlying localization regions. Therefore the algebraic properties above transfer to unions, causal complements and intersections of regions. We finally get

Theorem 2 Let $\mathcal{G}$ be the set of regions in $\mathbf{R}^{1,2}$ containing the wedges $W\left[l_{1}, l_{2}\right], W\left[l_{1}, l_{3}\right]$ and which is closed under

a) Lorentz boosting with $\Lambda_{12}(t), \Lambda_{13}(s)$,

b) intersection

c) (causal) union

d) translation in $l_{1}$ direction

e) causal complement

Then $\Delta_{W\left[l_{1}, l_{2}\right] \cap W\left[l_{1}, l_{3}\right]}^{i t}$ maps sets in $\mathcal{G}$ onto sets in $\mathcal{G}$ in a well computable way and extends the subgroup (49) by a "hidden symmetry".

Similarly we can look at a $(1+3)$-dim. quantum field theory. Then we get the same results as above for the modular theory to the region $W\left[l_{1}, l_{2}\right] \cap$ $W\left[l_{1}, l_{3}\right] \cap W\left[l_{1}, l_{4}\right]$, where $l_{i}$ are 4 linear independent lightlike vectors in $\mathbf{R}^{1,3}$. Moreover in this case the set $\mathcal{G}$ contain $W\left[l_{1}, l_{2}\right], W\left[l_{1}, l_{3}\right]$ and $W\left[l_{1}, l_{4}\right]$ and is closed under boosting with $\Lambda_{12}(t), \Lambda_{13}(s), \Lambda_{14}(r)$. 
The arguments are based on the Borchers commutation relation and modular theory and apply also if we replace modular intersection by modular inclusion. One recovers in this way the results of Borchers and Yngvason, Bo/Yng. ( Note that in thermal situations we have no simple geometrical interpretation for the commutants as the algebra to causal complements. Therefore in these cases we have to drop e) in the above theorem.).

The final upshot of this section is to show that there might be sensible meanings of geometrical actions of modular groups by restricting on certain subsystems.

\section{4-dim. Theories from a Finite Set of Alge- bras}

Let us mention a recent result due to Kähler and one of the authors (H.-W. W). It follows a line beginning with the work of Borchers, Bo1 and one of the authors, Wi2. Starting with a finite set of algebras lying in a specified position w.r.t. their common modular theory one constructs a net of local observables.

Theorem 3 Let $\mathcal{M}_{i j}, 0 \leq i<j \leq 4, \mathcal{M}_{i j}^{\prime}=\mathcal{M}_{j i}$, be von-Neumann algebras acting on $\mathcal{H}, \Omega \in \mathcal{H}$ with:

a) $\left(\mathcal{M}_{i j}, \mathcal{M}_{i k}, \Omega\right)$ has modular intersection

This part reflects the underlying wedge geometry.

b) symmetric in indices

This part means that there is no preferential "wedge" region. We can reformulate this as follows:

Let $\Gamma_{P}:=\Delta_{24}^{i t_{0}} \Delta_{34}^{-i t_{0}} \Delta_{32}^{i t_{0}} \Delta_{42}^{-i t_{0}}$ with:

b1) $\quad A d \Gamma_{P}\left(\mathcal{M}_{14}\right)=\mathcal{M}_{12}, \quad A d \Gamma_{P}\left(\mathcal{M}_{13}\right)=\mathcal{M}_{14}$

and $\Gamma_{P^{\prime}}:=\Delta_{13}^{i t_{0}} \Delta_{43}^{-i t_{0}} \Delta_{14}^{-i t_{0}} \Delta_{13}^{-t_{0}}$ with

$$
\text { Ad } \Gamma_{P^{\prime}}\left(\mathcal{M}_{12}\right)=\mathcal{M}_{32}, \quad \text { Ad } \Gamma_{P}\left(\mathcal{M}_{42}\right)=\mathcal{M}_{12}
$$


c) closed in some finite dim. Lie group

Essentially we use that the generators of the modular groups can be composed. We reformulate this as follows.

Denote $P^{1,1}$ the group generated by $\left\{\Delta_{14}^{i t_{14}} \Delta_{24}^{i t_{24}} \Delta_{34}^{i t_{34}}\right\}, S O(1,2)$ the one generated by $\left\{\Delta_{13}^{i t_{13}} \Delta_{12}^{i t_{12}} \Delta_{23}^{i t_{23}}\right\}$ Then let

$\left.c^{\prime}\right) \quad A d J_{12}\left(P_{\varepsilon}^{1,1}\right) \subset P_{\delta}^{1,1} \cdot S O(1,2), P_{\varepsilon}^{1,1}, \varepsilon-$ neighborhood of $1 \in P^{1,1}$

Then we conclude:

$$
\left\{\Delta_{14}^{i t_{14}}, \Delta_{24}^{i t_{24}}, \Delta_{34}^{i t_{34}}\right\},\left\{\Delta_{12}^{i t_{12}}, \Delta_{13}^{i t_{13}}, \Delta_{23}^{i t_{23}}\right\} \text { generate a reprs. of } \operatorname{Sl}(2 \mathbf{C}) .
$$

Proof. see $[\mathrm{Ka} / \mathrm{Wi}$

In order to get a representation of the Poincaré group we use the following. First we again implement rudimentarily the wedge geometry by:

Let $\mathcal{N}_{12}$ be a von-Neumann Algebra with

$$
\text { d) }\left(\mathcal{N}_{12} \subset \mathcal{M}_{12}, \Omega\right) \text { is hsm. }
$$

The resulting translations should be reflected by the CPT-like conjugations:

$$
\begin{aligned}
e 1) \quad A d J_{\mathcal{M}_{1 j}}\left(J_{\mathcal{N}_{12}} J_{\mathcal{M}_{12}}\right)=J_{\mathcal{M}_{12}} J_{\mathcal{N}_{12}}, & j=3,4 \\
e 2) \quad\left[\operatorname{Ad} J_{\mathcal{M}_{j k}}\left(J_{\mathcal{N}_{12}} J_{\mathcal{M}_{12}}\right), J_{\mathcal{N}_{12}} J_{\mathcal{M}_{12}}\right]=0, & j, k=2,3,4
\end{aligned}
$$

and the symmetry in the indices (no preferential wedge) leads to:

$$
\text { f) } \operatorname{Ad} \Gamma_{P}\left(J_{\mathcal{N}_{12}} J_{\mathcal{M}_{12}}\right)=J_{\mathcal{N}_{12}} J_{\mathcal{M}_{12}}
$$

Remark 1 Notice that $A d \Delta_{12}^{i t_{0}} \Delta_{13}^{-i t_{0}} \Gamma_{P}\left(\mathcal{M}_{12}\right)=\mathcal{M}_{12}$, so that f) implies

$\operatorname{Ad} \Delta_{12}^{i t_{0}} \Delta_{13}^{-i t_{0}} \Gamma_{P}\left(J_{\mathcal{N}_{12}}\right)=J_{\mathcal{N}_{12}}$. Modular theory then shows Ad $\Delta_{12}^{i t_{0}} \Delta_{13}^{-i t_{0}} \Gamma_{P}\left(\mathcal{N}_{12}\right)=$ $\mathcal{N}_{12}$.

Then we get a representation of $\mathbf{R}^{1,3}$, the translations.

The Lorentz group maps translations onto themselves which can be encoded in:

$$
\text { g) Ad } J_{j k}(\text { translations }) \subset \text { translations }
$$


Under these assumptions we get a representation of the full Poincaré group with spectrum condition. Using this one easily constructs a net of observables in $\mathbf{R}^{1,3}$ to it.

These results show that spacetime can be encoded in a finite set of algebraic data. Moreover spacetime is recovered by looking at the noncommutative structure measured w.r.t. the underlying physical state of the system (modular theory). This would not work in a classical, i.e. non (local) quantum case. Saying in an overstretched manner, the underlying classical geometry of spacetime results in our approach from the quantum theory by measuring the deviation from the commutative "classical" case. (Notice that by the "classical" case we do not refer to any underlying quantization procedure nor to a semiclassical approximation via some perturbation theory. The route we follow in our reconstruction of spacetime grounds in the noncommutativity and is intrinsically non pertubative.)

\section{On the modular theory of double cones (free massive case)}

Consider a double cone algebra $\mathcal{A}(\mathcal{O})$ generated by a free massless field (for $\mathrm{s}=0$ take the infrared convergent derivative). Then the modular objects of $(\mathcal{A}(\mathcal{O}), \Omega)_{m=0}$ are well-known Haa. In particular the modular group is a one parametric subgroup of the proper conformal group. The massive double cone algebra together with the (wrong) massless vacuum has the same modular group $\sigma_{t}$ however its action on smaller massive subalgebras inside the original one is not describable in terms of the previous subgroup. In fact the geometrical aspect of the action is wrecked by the breakdown of Huygens principle, which leads to a nonlocal reshuffling inside $O$ but still is local in the sense of keeping the inside and its causal complement apart. This mechanism can be shown to lead to a pseudo-differential operator for the infinitesimal generator of $\sigma_{t}$ whose's highest term still agrees with conformal zero mass differential operator. We are however interested in the modular group of $(\mathcal{A}(\mathcal{O}), \Omega)_{m}$ with the massive vacuum which is different from the that of the wrong vacuum by a Connes cocycle. We believe that this modular cocycle will not wreck the pseudo-differential nature, however we were not able to show this. We hope that the above remarks may prove helpful in a future investigation. 
ACKNOWLEDGMENT We would like to thank H.-J. Borchers and M. Schmidt for several discussions and remarks.

\section{References}

[Ar/Zs] H. Araki, L. Zsido "An Extension of the Structure Theorem of Borchers with an Application to Half-Sided Modular Inclusions" preliminary version (1995)

[Bi/Wi] J. Bisognano, E. Wichmann "On the Duality Condition for a Hermitean Scalar Field." Journ. Math. Physics 16 p. 985 (1975)

[Bo1] H.-J. Borchers "The CPT-Theorem in Two-dimensional Theories of Local Observables" Comm. Math. Phys. 143 p. 315 (1992)

[Bo2] H.-J. Borchers "Half-Sided Modular Inclusions and the Construction of the Poincaré Group " Comm. Math. Physics 176 p. 703 (1996

[Bo3] .-J. Borchers "On Poincaré transformations and the modular group of the algebra associated with a wedge" preprint (1998), to appear in Lett. Math. Phys.

[Bo4] H.-J. Borchers "When Does Lorentz Invariance Imply Wedge Duality" Lett. Math. Phys. 35 p. 39 (1995)

[Bo5] H.-J. Borchers "On the use of modular groups in quantum field theory" Ann. Henri Poincaré 63 p 331 (1995).

[Bo/Yng] H.-J. Borchers, J. Yngvason "Modular Groups of Quantum Fields in Thermal States." preprint (1998)

[Fr/Joe] K. Fredenhagen and Joerss, "Conformal Haag-Kastler nets, pointlike localized fields and the existence of operator product expansions", Comm. Math. Phys. 176, (1996) p. 541

[B/D/F/S] D. Buchholz, O. Dreyer, M. Florig and S. Summers, "Geometric Modular Action and Spacetime Symmetry Groups" hep 9805026 
[G/L/W] D. Guido, R. Longo, H.-W. Wiesbrock "Extensions of Conformal Nets and Superselection Structures" Comm. Math.Phys. 192 p.217 (1998)

[Haa] R. Haag "Local Quantum Physics" Springer Verlag (1992)

$[\mathrm{H} / \mathrm{H} / \mathrm{W}] \quad$ R. Haag, N. Hugenholtz, M. Winnink "On the equilibrium state in quantum statistical mechanics" Comm. Math. Phys. 5 p 215 (1967)

[Ja] Ch. Jaekel "Cluster Estimates for Modular Structure" hep 9804017

[Ka/Wi] R. Kaehler, H.-W. Wiesbrock "Modular Theory and the Reconstruction of 4-dim. Quantum field theories" in preparation

[Pe] G. Pedersen "C*-algebras and their automorphism groups" Academic Press, London, New York, San Francisco (1979)

[O/L/R] P. Leyland, J. Roberts and D. Testard, "Duality for Quantum Fields" unpublished preprint July 1978

J. P. Eckmann and K. Osterwalder, J. Funct. An. 13, (1973) 1

[Schr1] B. Schroer, "Motivations and Physical Aims of algebraic Quantum Field Theory" Amm. Phys. (N.Y.) 255, (1997) 270. The relation between the positive energy and the isotony of the net of wedge subspaces of the Wigner representation one of the authors (B. S.) learned from a talk by D. Guido 1996.

[Schr2] B. Schroer, "Modular Wedge Localization and the $d=1+1$ Formfactor Program" hep-th/9712124, to be published in AOP.

[Schr3] B. Schroer, a course on "Localization and Nonperturbative Local Quantum Physics", hep-th/9805093, chapter 3 and 6

[Se] G. Sewell "Quantum Fields on Manifolds: PCT and Gravitationally Induced Thermal States" Ann. of Phys. 141 p 201 (1982)

[Stra] S. Stratila "Modular Theory in Operator Algebras" Abacus Press 1981. 
[Ta] M. Takesaki "Tomita's Theory of Modular Hilbert Algebras and its Applications" Lect. Notes in Math. 128 Springer Verlag (1970)

[Un] W. Unruh "Notes on Black Hole Evaporation" Phys. Rev. D29 p 1047 (1976)

[Was] A. Wassermann, "Operator Algebras and Conformal Field Theory", University of Cambridge preprint 1997

[Wi1] H.-W. Wiesbrock "Half-Sided Modular Inclusions of vonNeumann Algebras" Comm. Math. Phys. 157 p 83 (1993) Erratum Comm. Math. Phys. 184 p.683 (1997)

[Wi2] H.-W. Wiesbrock "Conformal Quantum Field Theory and HalfSided Modular Inclusions of von-Neumann Algebras." Comm. Math. Phys. 158 p 537 (1993)

[Wi3] H.-W. Wiesbrock "Symmetries and Modular Intersections of vonNeumann Algebras" Lett. Math. Phys. 39 p 203 (1997)

[Wi4] H.-W. Wiesbrock "Modular Intersections of von-Neumann Algebras in Quantum Field Theory" Comm. Math. Phys. 193 p 269 (1998)

[Yng] J. Yngvason "A Note on Essential Duality" Lett. Math. Phys. 31 p 127 (1995) 